\title{
Biomarkers in Multiple Sclerosis: a review of diagnostic and prognostic factors
}

\author{
Klaudia Sapko, Anna Jamroz-Wiśniewska, Michał Marciniec, Marcin Kulczyński, \\ Anna Szczepańska-Szerej, Konrad Rejdak \\ Chair and Department of Neurology, Medical University of Lublin, Poland
}

\begin{abstract}
Introduction. Multiple Sclerosis (MS) is a chronic, demyelinating disease of the central nervous system which affects mostly young people. Because it leads to disability and cognitive impairment, it is crucial to recognise MS at an early stage.

State of the art. Magnetic resonance imaging is the golden standard in MS diagnosis. However, it is not an infallible diagnostic tool, especially at the stage of clinically isolated syndrome. The incorporation of oligoclonal bands in the diagnostic process of MS is a step towards the extension of diagnostic methods. Recently, a lot of research has been carried out on potential biomarkers in blood serum and cerebrospinal fluid that may be useful in the diagnosis of MS.

Clinical implications. This article summarises current knowledge on the use of new prognostic factors such as neurofilament light chain, chitinase 3-like 1 and 2, heat shock proteins, and tubulins in MS.

Future directions. Despite numerous studies on the use of biomarkers in the diagnosis of MS, more extensive research is needed to determine the clinical usefulness of these molecules and to develop diagnostic tests applicable in everyday practice. This in turn may result in earlier MS detection, faster implementation of treatment, and better therapeutic effects.

Key words: multiple sclerosis, biomarker, risk factor, cerebrospinal fluid, serum

(Neurol Neurochir Pol 2020; 54 (3): 252-258)
\end{abstract}

\section{Introduction}

Multiple Sclerosis (MS) is a chronic, inflammatory, demyelinating disease of the central nervous system (CNS) with a wide spectrum of clinical and imaging changes $[1,2]$. The first episode of neurological symptoms with the features of inflammatory demyelination is referred to as clinically isolated syndrome (CIS) [3]. This develops acutely or subacutely, lasts at least 24 hours, and runs a course similar to that of a typical MS relapse but in a patient not yet diagnosed with MS $[3,4]$. Research has shown that up to $60-70 \%$ of patients with a first clinical episode do not meet the criteria for MS. However, up to $85 \%$ of these patients will develop full-blown MS in the future $[5,6]$.

According to the new, revised McDonald criteria from 2017, magnetic resonance imaging (MRI) is the golden standard in MS diagnosis [7]. The risk of conversion from CIS to clinically definite MS (CDMS) in patients with characteristic lesions in MRI is up to 60-80\%, while in patients without abnormalities specific for MS this risk is $20 \%$ at most $[3,8,9]$. Unfortunately, MRI is not effective enough to detect all active demyelinating changes in the CNS. MS affects approximately 2.3 million people, mostly between the ages of 20 and 40 , leading to their disability and cognitive impairment. This makes it a significant problem for society as a whole [10]. Currently available treatment can delay the progression of MS only if it is used at the beginning of the disease. This is why early diagnosis of MS has become a burning clinical challenge. It is essential to properly recognise it from the very earliest stages of the disease. The above-mentioned McDonald criteria put emphasis on the early detection of the CIS. In addition to magnetic resonance, oligoclonal bands (OCBs) play a key role in the diagnostic process. Moreover, recent promising studies have been carried out to determine

Address for correspondence: Klaudia Sapko, Chair and Department of Neurology, Medical University of Lublin, Jaczewskiego 8, 20-954 Lublin, Poland, e-mail: klaudia.sapko@gmail.com 


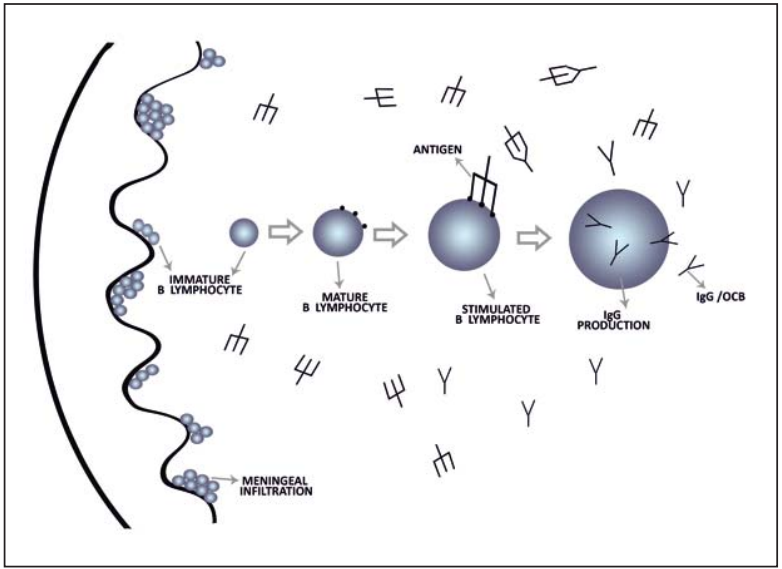

Figure 1. Intrathecal synthesis of immunoglobulins $G$ forming oligoclonal bands. Immature B lymphocytes form infiltrates meninges, where they go through maturation process and acquire affinity to antigen. Mature B lymphocytes become stimulated B lymphocytes after contact with antigen. Stimulated B lymphocytes start producing immunoglobulins $\mathrm{G}$, which form oligoclonal bands; lgG - immunoglobulin G; OCB - oligoclonal band

the presence and concentration of new biochemical indicators in the cerebrospinal fluid (CSF) and blood serum that may be useful in MS diagnosis.

\section{State of the art}

\section{Oligoclonal bands as predictive factors of MS}

OCBs are mostly immunoglobulins $\mathrm{G}(\mathrm{IgG})$, which are produced intrathecally by stimulated clones of $B$ lymphocytes and plasma cells. It is believed that the final maturation of $B$ lymphocytes, and their affinity for a closer unknown antigen, occurs in the peritoneum and meninges (Fig. 1) [11, 12]. CSF and the serum are tested by isoelectric focusing (IEF) gel electrophoresis for the presence of so-called bands. There are six types of OCBs: types 2 and 3 indicate intrathecal synthesis; the other four are negative for MS (Tab. 1) [13-15].

The importance of oligoclonal bands in the diagnosis of MS has been the subject of several studies. A large study conducted by 33 centres included 1,047 patients with CIS observed for a minimum of two years. With a median follow-up of 4.31 years, 623 patients with CIS converted to MS. Oligoclonal bands were detected in $95 \%$ of patients [6].

Another multicentre retrospective study, conducted by German-Austrian scientists, included 406 patients. The diagnostic criteria for MS were met by $11 \%$ of patients ( 44 people), and the remaining $89 \%$ (362 people) were diagnosed with CIS. OCBs were positive in $86 \%$ of patients with CIS; $74 \%$ of these patients developed MS. OCBs were not detected in $14 \%$ of patients; $44 \%$ of these patients converted to MS. The median conversion time for CIS patients with positive OCBs was 25 months, compared to 47 months in patients with negative OCBs [16].
Table 1. Types of OCBs depending on their pattern in IEF and occurrence in body fluids [13]

\section{IEF pattern Occurrence in body fluids}

$1 \quad$ No OCBs in CSF and serum

2

3

CSF-restricted OCBs and additional identical bands in CSF and serum (combination of patterns 2 and 4 )

4 Identical OCBs in CSF and serum ('mirror pattern')

5 Monoclonal bands in CSF and serum

6 Presence of a single band limited to CSF

OCBs - oligoclonal bands; IEF — isoelectric focusing; CSF — cerebrospinal fluid; IgG — immunoglobulin $\mathrm{G}$

Another study included 120 patients with CIS. Conversion to MS was observed in $42 \%$ of cases, and $58 \%$ of patients was defined as stable CIS. Positive OCBs were detected in $61 \%$ of patients with CIS at the beginning of the study. During followup, $55 \%$ of patients with positive OCBs converted to MS, and $21 \%$ of patients with negative OCBs developed MS. The median time needed for conversion was similar in both groups [17]. These two studies show that people with CIS and positive test results for OCBs are twice as likely to develop MS than people with negative OCBs. However, in the German-Austrian study, the conversion time to MS was almost twice as long in patients with negative OCBs than it was in patients with positive OCBs.

Recent research has also focused on the value of intrathecal immunoglobulin M (IgM) synthesis in the diagnosis of MS. A study involving 126 patients with CIS showed that IgM levels have a higher reliability index for the conversion of patients with CIS to CDMS compared to IgG [18].

Generally, oligoclonal bands are a strong independent predictor of the risk of conversion in patients with CIS to CDMS: this has been proven by numerous studies. Based on this, an oligoclonal bands test was included in the latest (2017) McDonald criteria, not only as a criterion of MS but also as a predictor for a second relapse occurrence in patients diagnosed with CIS [7]. Moreover, OCBs are not specific indicators for MS, because an increased level of OCBs can occur in other infectious and inflammatory diseases of the central nervous system $[19,20]$.

\section{New potential biochemical indicators of MS}

\section{Neurofilament light chain}

Neurofilaments (NFs) are larger molecules of the neuronal cytoskeleton. They are divided into three subgroups: light, medium, and heavy [21]. Neurofilaments are neuron-specific proteins that are released during neuronal damage. This process is the main element of pathology in MS. Therefore it may result in the occurrence of neurofilament light chain (NFL) in the cerebrospinal fluid and then in the blood serum [22-24]. It is believed that axonal damage occurs in the early stages of MS [25, 26]. 
The role of the neurofilament light chain as a diagnostic factor in MS has been the subject of much research. A prospective longitudinal cohort study included 41 patients with CIS or relapsing-remitting multiple sclerosis (RRMS) and 22 healthy patients. Patients' cerebrospinal fluid was analysed for many biomarkers, including neurofilament light chain. In addition, during the two-year follow-up, the activity of the disease was evaluated by assessing relapses, worsening of disability, or magnetic resonance imaging activity. The study showed that NFL turned out to be the best predictor of MS development at the baseline. Within two years of follow-up, based on the NFL level, $93 \%$ of patients who showed signs of disease activity, as well as $67 \%$ of patients who did not, were correctly classified. The overall percentage of correct classifications was $85 \%$ (33/39 patients) [27].

Another study included 85 RRMS patients whose serum was collected and tested for NFL and its potential role as a predictor of disease activity. Patients were followed for two years. They did not receive disease-modifying treatment for the first six months, then for the next 18 months they received interferon-beta 1a (IFNB-1a). Baseline assessment included the collection of serum samples, MRI and the Expanded Disability Status Scale (EDSS). Serum samples were additionally collected after three, six, 12 and 24 months, MRI was performed after nine, 12 and 24 months and EDSS was evaluated every six months. Patients with new T1 gadolinium-enhancing lesions and new T2 lesions had significantly higher serum NFL levels compared to patients without new changes in MRI. The presence of T1 gadolinium-enhancing lesions was correlated with serum NFL levels two months before and one month after biochemical measurements. The level of NFL decreased after inclusion of IFNB-1a treatment [28].

A third study was carried out on 86 patients with optic neuritis $(\mathrm{ON})$ as the first manifestation of demyelination. The level of neurofilament light chain was examined in the cerebrospinal fluid. Patients were followed on average for 13.6 years, and $81.4 \%$ of patients were evaluated using MRI and EDSS scores. The remaining $18.6 \%$ of patients were questioned by telephone. During follow-up, 53.5\% (46 patients) developed CDMS, and NFL predicted long-term disability by the multiple sclerosis severity scale [29].

The last study was performed on 75 patients with radiologically isolated syndrome (RIS). The level of NFL was measured in cerebrospinal fluid. Neurofilament light chain was an independent risk factor for conversion to a CIS and MS. A high level of NFL was associated with a tendency to shorter conversion time to CIS, and much shorter to CDMS, which was more evident in RIS patients aged at least 37 years compared to younger patients [30].

In summary, the NFL level in the cerebrospinal fluid in patients with CIS and RRMS appears to be a strong potential prognostic factor in the assessment of disease activity [27, 31]. Serum NFL is a promising biochemical indicator for the effects of treatment in RRMS and an alternative to MRI in the assessment of subclinical disease activity [28, 32, 33]. Moreover, NFL turned out to be a predictor of long-term physical and cognitive disability after optic neuritis as the first demyelinating manifestation, and an independent risk factor for conversion to CIS and CDMS in patients with RIS [29, 30].

\section{Chitinase 3-like 1(Human YKL-40, CHI3L1) and chitinase 3-like 2 (Human CHI3L2)}

Chitinase 3-like 1 (Human YKL-40, CHI3L1) and chitinase 3-like 2 (Human CHI3L2) are produced by neutrophils, astrocytes and macrophages as enzymatically inactive proteins which are involved in tissue remodelling and inflammation [34]. The levels of these substances increase in the cerebrospinal fluid during various acute and chronic neuroinflammatory states, including MS [35].

One of the first studies to determine the usefulness of chitinase 3-like 1 in the diagnosis of MS was a study performed on a group of 60 CIS patients, 30 of whom underwent conversion to CDMS, while the remaining 30 patients were stable CIS.

Analysis of patient cerebrospinal fluid revealed a significant correlation between the high level of CHI3L1 and the number of gadolinium-enhancing lesions as well as the number of T2 lesions in MRI at the beginning of the study, and was associated with a faster progression of disability and shorter conversion time to CDMS. The level of chitinase 3-like 1 was also higher among patients who underwent conversion to MS compared to those who remained stable CIS [36].

The above results have been confirmed in another longitudinal cohort study carried out in 15 European MS centres. A total of 813 cerebrospinal fluid samples were collected from patients with a CIS. The CHI3L1 level was higher in patients who converted to CDMS compared to those who continued treatment as a CIS. A high level of CHI3L1 was also associated with a shorter conversion time to MS and faster development of disability [37].

Another study investigated the relationship between CHI3L1 levels and response to interferon-beta (IFN $\beta$ ) and glatiramer acetate (GA) in patients with MS. The level of CHI3L1 in the cerebrospinal fluid of 117 patients with RRMS was measured, including 76 patients treated with IFN $\beta$ and 41 treated with GA. The level of CHI3L1 was associated with the response to INF $\beta$ treatment, and was higher in the group of non-responders. Similar effects were not found among patients treated with GA. CHI3L1 may thus act as a response biomarker to INF $\beta$ in patients with RRMS [38].

Chitinase 3-like 1 can be used as a biomarker of disability development and as an effective marker to distinguish patients who will convert to CDMS from those who will remain as stable CIS in the future $[36,37]$. CHI3L1 is also a potential biomarker for response to INF $\beta$ treatment in patients with RRMS [38].

On the other hand, the potential of chitinase 3 -like 2 in the diagnosis of MS was examined in a prospective cohort study which included 73 patients with optic neuritis as the first demyelinating event, plus 26 age-matched healthy subjects. The 
level of CHI3L2 was determined in the cerebrospinal fluid. The predictive capacity of CHI3L2 was compared to that of CHI3L1. The level of CHI3L2 was significantly elevated in patients with $\mathrm{ON}$ and was associated with the risk of developing MS. In addition, CHI3L2 was correlated with the risk of cognitive impairment and MS development in patients after ON.

CHI3L2 is a promising risk factor in patients with the first episode of demyelination. In multifactorial risk analysis of MS, CHI3L2 has been shown to be more effective than CHI3L1 [39].

\section{Heat shock protein 70 and heat shock protein 90}

Heat shock proteins (HSPs) are molecular chaperones that play vital homeostatic roles in the central nervous system and whose distribution between different species is conservative. They can be divided into different groups depending on the molecular weight [40]. Heat shock protein 70 (HSP70) is localised in the cytosol, where it supports and protects cells against lethal stress-induced damage, as well as in the cell membrane and the intracellular space, where it plays an important role in the immune response [41, 42]. Elevated levels of HSP70 can have a beneficial effect on MS, protecting neurons and oligodendrocytes during an inflammatory process from death through apoptosis. However, the extracellular HSP70 may be responsible for induction of immunologic reaction $[43,44]$.

The first study was based on analysis of the HSPA1L gene polymorphism encoding the HSP70-hom protein among 191 MS patients and 365 healthy subjects. There was a strong correlation between the polymorphism of the studied gene and the risk of MS development, as well as a significant relationship between the expression of the HSP70-hom protein and the severity of MS [45].

The purpose of further research was to determine the role of HSP70 as a potential biomarker in the differentiation of neurodegenerative and inflammatory processes in MS. The serum of 94 patients with MS was examined, including 26 with CIS, 40 with RRMS, 19 with secondary progressive MS (SPMS), and nine with primary progressive MS (PPMS). The control group consisted of 41 patients with non-inflammatory neurological diseases (NIND), 28 with other inflammatory neurological diseases (OIND), and 114 healthy donors (HD). The level of HSP70 in the serum of patients with MS was significantly higher than in HD, and significantly lower than in OINDs. Moreover, the analysis showed that the HSP70 level in patients with CIS or RRMS was significantly higher than in patients with PPMS or SPMS, which may be correlated with the strongly expressed inflammatory process in the first group [46].

HSP70 is considered to be a useful biomarker to monitor inflammatory processes in MS in the future $[45,46]$. Nevertheless, there is still no consensus as to whether HSP70 mediates the beneficial or negative effects of MS.

Heat shock protein 90 (HSP90) has similar properties to HSP70, differing only in molecular weight. It modulates inflammatory processes by producing anti-inflammatory cytokines and modulating the response with toll-like receptor 2 and 4 (TLR2 and TLR4) [47].

One study evaluated the effect of HSP90 on steroid response in the treatment of relapses in MS patients. It was shown that the amount of HSP90 in the glucocorticoid receptor (GR) complex was significantly higher in patients with steroid-resistant MS than in patients with steroid-sensitive MS. The mechanism of non-response to glucocorticoids may be associated with an increased presence of HSP90 in the cytoplasmic GR complex, which causes inhibition of GR translocation to the nucleus and reduction of its transcription [48].

\section{Tubulin beta (TUBß)}

Tubulins (TUBs) are heterodimeric proteins consisting of an alpha and a beta subunit and are major components of microtubules. The synthesis of class II tubulin isotype increases in development and regeneration of neurons.

In one study, the level of cytoskeletal proteins, including tubulin beta, in the cerebrospinal fluid of patients with MS and their correlation with clinical indicators of MS was assessed. The study was performed in 51 patients, including 33 with MS and 18 with other neurological diseases (OND). Tubulin beta (TUB $\beta$ ) level was significantly higher in MS patients than in OND patients [49]. Preliminary results show that TUB $\beta$ is a promising diagnostic factor in MS, but further analyses are needed.

\section{Combined measurements of biochemical indicators and their impact on the diagnosis of $M S$}

Recently, studies have been conducted to determine the effect of combined measurements of some of the above-discussed biomarkers levels in CSF and their role in the diagnosis of MS.

In a cross-sectional cohort study by Spanish researchers, the correlation between NFL and CHI3L1 levels in CSF in 157 MS patients, including 99 patients with RRMS, 35 with SPMS, and 23 with PPMS, was investigated. After 50 months of observation, it was found that NFL and CHI3L1 levels in CSF were higher in patients with MS compared to patients in the disease-free control group. Increased levels of NFL in RRMS and SPMS patients were characteristic of clinical relapse, while high CHI3L1 levels were associated with progressive disease. NFL and CHI3L1 levels correlated with each other and with IgM-oligoclonal bands in RRMS patients. A formula of combined measurements of biomarkers was useful in determining MS phenotypes and in predicting clinical progression. High NFL and low CHI3L1 levels occur more frequently in RRMS compared to SPMS and PPM. In turn, elevated levels of both biomarkers were ahead of diagnosis of clinical progression in patients with RRMS [50].

A second study tracked the diagnostic value of NFL and CHI3L1 levels in CSF in 177 newly diagnosed patients with CIS or RRMS. Patients were clinically followed for an average 
Table 2. Usefulness of prognostic biomarkers in diagnosis of different types and stages of MS

\begin{tabular}{|c|c|c|c|c|c|c|}
\hline & CIS & RIS & ON & RRMS & CDMS & S-R MS \\
\hline NFL & + & + & + & + & + & - \\
\hline CHI3L1 & + & - & - & + & + & - \\
\hline CHI3L2 & - & - & + & - & - & - \\
\hline HSP70 & + & - & - & + & - & - \\
\hline HSP9O & - & - & - & - & - & + \\
\hline TUB $\beta$ & - & - & - & - & + & - \\
\hline
\end{tabular}

$\mathrm{NFL}$ - neurofilament light chain; CHI3L1 - chitinase 3-like 1; CHI3L2 - chitinase 3-like 2; HSP70 - heat shock protein 70; HSP90 - heat shock protein 90; TUBß - tubulin beta; CIS - clinically isolated syndrome; RIS — radiologically isolated syndrome; ON — optic neuritis; RRMS - relapsing-remitting multiple sclerosis; CDMS — clinically defined multiple sclerosis; S-R MS — steroid-resistant multiple sclerosis; $(+)$ useful in diagnostics; $(-)$ not useful in diagnostics

of 5.7 years. Both NFL and CHI3L1 concentrations in CSF were associated with a higher risk of relapse during the first two years in one dimensional analyses, in contrast to multivariable analysis where only the NFL level was associated with relapse risk. No relationship was found between NFL or CHI3L1 concentrations and risk of conversion to SPMS or disability progression [51].

The research we have described shows that combined measurements of new biochemical indicators such as NFL and CHI3L1 levels in CSF may bring benefits in the diagnosis and prognosis of MS and may set a new direction for research using other biomarkers.

\section{Clinical implications}

MS is a disease that affects mainly young people and leads to their disability, which is why it is so important to diagnose it as early as possible. The insufficient accuracy of MRI in early MS diagnosis has led to a search for new predictors. Determining the OCBs level in the cerebrospinal fluid is included in the latest McDonald's criteria for the diagnosis of MS. Recently, promising studies have been carried out on potential new biochemical markers in the blood serum and cerebrospinal fluid that may be useful in the diagnosis of different forms and stages of MS (Tab. 2). The first results of research on compounds such as neurofilament light chain, chitinase 3-like 1 and 2 , heat shock proteins, tubulins, or combined measurements of some of these biomarkers, bring fresh hope for patients and for doctors seeking to diagnose MS.

\section{Future directions}

Despite numerous studies on the use of biomarkers in the diagnosis of MS, more extensive research is needed to determine the clinical usefulness of these molecules. Identification of sensitive and specific biomarkers in CSF and blood serum of patients with CIS, the development of standardised diagnostic tests detecting these markers, and their use in everyday clinical practice, may result in earlier MS detection, faster implementation of treatment, and better therapeutic effects.
Ethical approval. Was not necessary for the preparation of this article.

Funding. This publication was prepared without any external source of funding.

Conflicts of interest. The authors declare that they have no conflict of interest.

\section{References}

1. Kantarci $\mathrm{OH}$, Weinshenker BG. Natural history of multiple sclerosis. Neurol Clin. 2005; 23: 17-38, doi: 10.1016/j.ncl.2004.10.002.

2. Rejdak K, Jackson S, Giovannoni G. Multiple sclerosis: a practical overview for clinicians. Br Med Bull. 2010; 95: 79-104, doi: 10.1093/ bmb/ldq017, indexed in Pubmed: 20603280.

3. Efendi H. Clinically Isolated Syndromes: Clinical Characteristics, Differential Diagnosis, and Management. Noro Psikiyatr Ars. 2015; 52 : 1-11, doi: 10.5152/npa.2015.12608.

4. Miller D, Barkhof F, Montalban X, et al. Clinically isolated syndromes suggestive of multiple sclerosis, part I: natural history, pathogenesis, diagnosis, and prognosis. Lancet Neurol. 2005; 4: 281-288, doi: 10.1016/S1474-4422 (05) 70071-5.

5. Miller DH, Chard DT, Ciccarelli O. Clinically isolated syndromes. Lancet Neurol. 2012; 11(2): 157-169, doi: 10.1016/S1474-4422(11)702745, indexed in Pubmed: 22265211.

6. Kuhle J, Disanto G, Dobson R, et al. Conversion from clinically isolated syndrome to multiple sclerosis: A large multicentre study. Mult Scler. 2015; 21(8): 1013-1024, doi: 10.1177/1352458514568827, indexed in Pubmed: 25680984.

7. Thompson AJ, Banwell BL, Barkhof F, et al. Diagnosis of multiple sclerosis: 2017 revisions of the McDonald criteria. Lancet Neurol. 2018; 17(2): 162-173, doi: 10.1016/S1474-4422(17)30470-2, indexed in Pubmed: 29275977.

8. Gaetani L, Fanelli F, Riccucci I, et al. High risk of early conversion to multiple sclerosis in clinically isolated syndromes with dissemination in space at baseline. J Neurol Sci. 2017; 379: 236-240, doi: 10.1016/j.jns.2017.06.008, indexed in Pubmed: 28716249.

9. Marcus JF, Waubant EL. Updates on clinically isolated syndrome and diagnostic criteria for multiple sclerosis. Neurohospitalist. 2013; 3(2): 65-80, doi: 10.1177/1941874412457183, indexed in Pubmed: 23983889.

10. Brownlee WJ, Hardy TA, Fazekas F, et al. Diagnosis of multiple sclerosis: progress and challenges. Lancet. 2017; 389(10076): 13361346, doi: 10.1016/S0140-6736(16)30959-X, indexed in Pubmed: 27889190 . 
11. Bankoti J, Apeltsin L, Hauser SL, et al. In multiple sclerosis, oligoclonal bands connect to peripheral B-cell responses. Ann Neurol. 2014; 75(2): 266-276, doi: 10.1002/ana.24088, indexed in Pubmed: 24375699.

12. Serafini B, Rosicarelli B, Magliozzi R, et al. Detection of ectopic B-cell follicles with germinal centers in the meninges of patients with secondary progressive multiple sclerosis. Brain Pathol. 2004; 14(2): 164-174, doi: 10.1111/j.1750-3639.2004.tb00049.x, indexed in Pubmed: 15193029.

13. Dobson R, Ramagopalan S, Davis A, et al. Cerebrospinal fluid oligocIonal bands in multiple sclerosis and clinically isolated syndromes: a meta-analysis of prevalence, prognosis and effect of latitude. J Neurol Neurosurg Psychiatry. 2013; 84(8): 909-914, doi: 10.1136/ jnnp-2012-304695, indexed in Pubmed: 23431079.

14. Jarius S, König FB, Metz I, et al. Pattern II and pattern III MS are entities distinct from pattern I MS: evidence from cerebrospinal fluid analysis. J Neuroinflammation. 2017; 14(1): 171, doi: 10.1186/ s12974-017-0929-z, indexed in Pubmed: 28851393.

15. Pinar A, Kurne AT, Lay I, et al. Cerebrospinal fluid oligoclonal banding patterns and intrathecal immunoglobulin synthesis: Data comparison from a wide patient group. Neurological Sciences and Neurophysiology. 2018; 35(1): 21-28, doi: 10.5152/nsn.2018.10247.

16. Huss AM, Halbgebauer S, Öckl P, et al. Importance of cerebrospinal fluid analysis in the era of McDonald 2010 criteria: a German-Austrian retrospective multicenter study in patients with a clinically isolated syndrome. J Neurol. 2016; 263(12): 2499-2504, doi: 10.1007/ s00415-016-8302-1, indexed in Pubmed: 27730374.

17. Schwenkenbecher P, Sarikidi A, Bönig L, et al. Clinically Isolated Syndrome According to McDonald 2010: Intrathecal IgG Synthesis Still Predictive for Conversion to Multiple Sclerosis. Int J Mol Sci. 2017; 18(10), doi: 10.3390/ijms18102061, indexed in Pubmed: 28953254.

18. Huss A, Abdelhak A, Halbgebauer $S$, et al. Intrathecal immunoglobulin M production: A promising high-risk marker in clinically isolated syndrome patients. Ann Neurol. 2018; 83(5): 1032-1036, doi: 10.1002/ ana.25237, indexed in Pubmed: 29665046.

19. Ferraro D, Galli V, Simone AM, et al. Cerebrospinal fluid anti-Epstein-Barr virus specific oligoclonal IgM and lgG bands in patients with clinically isolated and Guillain-Barré syndrome. J Neurovirol. 2017; 23(2): 329-334, doi: 10.1007/s13365-016-0493-9, indexed in Pubmed: 27878471.

20. Schwenkenbecher P, Pul R, Wurster U, et al. Common and uncommon neurological manifestations of neuroborreliosis leading to hospitalization. BMC Infect Dis. 2017; 17(1): 90, doi: 10.1186/s12879-0162112-z, indexed in Pubmed: 28109263.

21. Khalil M, Salzer J. CSF neurofilament light: A universal risk biomarker in multiple sclerosis? Neurology. 2016; 87(11): 1068-1069, doi: 10.1212/WNL.00000000000003107, indexed in Pubmed: 27521432.

22. Gnanapavan S, Grant D, Morant S, et al. Biomarker report from the phase II lamotrigine trial in secondary progressive MS - neurofilament as a surrogate of disease progression. PLoS One. 2013; 8(8): e70019, doi: 10.1371/journal.pone.0070019, indexed in Pubmed: 23936370.

23. Gunnarsson M, Malmeström C, Axelsson M, et al. Neurofilament and glial fibrillary acidic protein in multiple sclerosis. Neurology. 2004; 63(9): 1586-1590, doi: 10.1212/01.wnl.0000142988.49341.d1, indexed in Pubmed: 15534240.

24. Soelberg Sorensen P, Sellebjerg F. Neurofilament in CSF-A biomarker of disease activity and long-term prognosis in multiple sclerosis. Mult Scler. 2016; 22(9): 1112-1113, doi: 10.1177/1352458516658560, indexed in Pubmed: 27364323.
25. Kuhlmann T, Lingfeld G, Bitsch A, et al. Acute axonal damage in multiple sclerosis is most extensive in early disease stages and decreases over time. Brain. 2002; 125(Pt 10): 2202-2212, doi: 10.1093/brain/ awf235, indexed in Pubmed: 12244078.

26. Disanto G, Barro C, Benkert P, et al. Swiss Multiple Sclerosis Cohort Study Group. Serum Neurofilament light: A biomarker of neuronal damage in multiple sclerosis. Ann Neurol. 2017; 81(6): 857-870, doi: 10.1002/ana.24954, indexed in Pubmed: 28512753.

27. Håkansson I, Tisell A, Cassel P, et al. Neurofilament light chain in cerebrospinal fluid and prediction of disease activity in clinically isolated syndrome and relapsing-remitting multiple sclerosis. Eur J Neurol. 2017; 24(5): 703-712, doi: 10.1111/ene.13274, indexed in Pubmed: 28261960.

28. Varhaug KN, Barro C, Bjørnevik K, et al. Neurofilament light chain predicts disease activity in relapsing-remitting MS. Neurol Neuroimmunol Neuroinflamm. 2018; 5(1): e422, doi: 10.1212/ NXI.0000000000000422, indexed in Pubmed: 29209636.

29. Modvig S, Degn M, Sander B, et al. Cerebrospinal fluid levels of chitinase 3 -like 1 and neurofilament light chain predict multiple sclerosis development and disability after optic neuritis. Mult Scler. 2015; 21(14): 1761-1770, doi: 10.1177/1352458515574148, indexed in Pubmed: 25698172.

30. Matute-Blanch C, Villar LM, Álvarez-Cermeño JC, et al. Neurofilament light chain and oligoclonal bands are prognostic biomarkers in radiologically isolated syndrome. Brain. 2018; 141(4): 1085-1093, doi: 10.1093/brain/awy021, indexed in Pubmed: 29452342.

31. Bhan A, Jacobsen C, Myhr KM, et al. Neurofilaments and 10-year follow-up in multiple sclerosis. Mult Scler. 2018; 24(10): 1301-1307, doi: 10.1177/1352458518782005, indexed in Pubmed: 30066611.

32. Kuhle J, Barro C, Disanto G, et al. Serum neurofilament light chain in early relapsing remitting MS is increased and correlates with CSF levels and with MRI measures of disease severity. Mult Scler. 2016; 22(12): 1550-1559, doi: $10.1177 / 1352458515623365$, indexed in Pubmed: 26754800.

33. Novakova L, Zetterberg $\mathrm{H}$, Sundström $\mathrm{P}$, et al. Monitoring disease activity in multiple sclerosis using serum neurofilament light protein. Neurology. 2017; 89(22): 2230-2237, doi: 10.1212/ WNL.0000000000004683, indexed in Pubmed: 29079686.

34. Bonneh-Barkay D, Bissel SJ, Kofler J, et al. Astrocyte and macrophage regulation of YKL-40 expression and cellular response in neuroinflammation. Brain Pathol. 2012; 22(4): 530-546, doi: 10.1111/j.1750-3639.2011.00550.x, indexed in Pubmed: 22074331.

35. Bonneh-Barkay D, Wang G, Starkey A, et al. In vivo CHI3L1 (YKL-40) expression in astrocytes in acute and chronic neurological diseases. J Neuroinflammation. 2010; 7: 34, doi: 10.1186/1742-2094-7-34, indexed in Pubmed: 20540736.

36. Comabella M, Fernández M, Martin R, et al. Cerebrospinal fluid chitinase 3-like 1 levels are associated with conversion to multiple sclerosis. Brain. 2010; 133(Pt 4): 1082-1093, doi: 10.1093/brain/ awq035, indexed in Pubmed: 20237129.

37. Cantó E, Tintoré M, Villar LM, et al. Chitinase 3-like 1: prognostic biomarker in clinically isolated syndromes. Brain. 2015; 138(Pt 4): 918931, doi: 10.1093/brain/awv017, indexed in Pubmed: 25688078.

38. Matute-Blanch C, Río J, Villar LM, et al. Chitinase 3-like 1 is associated with the response to interferon-beta treatment in multiple sclerosis. J Neuroimmunol. 2017; 303: 62-65, doi: 10.1016/j.jneuroim.2016.12.006, indexed in Pubmed: 28063616.

39. Møllgaard M, Degn M, Sellebjerg F, et al. Cerebrospinal fluid chitinase-3-like 2 and chitotriosidase are potential progno- 
stic biomarkers in early multiple sclerosis. Eur J Neurol. 2016; 23(5): 898-905, doi: 10.1111/ene.12960, indexed in Pubmed: 26872061.

40. Daugaard M, Rohde M, Jäättelä M. The heat shock protein 70 family: Highly homologous proteins with overlapping and distinct functions. FEBS Lett. 2007; 581(19): 3702-3710, doi: 10.1016/j.febslet.2007.05.039, indexed in Pubmed: 17544402.

41. Pockley AG, Henderson B, Multhoff G. Extracellular cell stress proteins as biomarkers of human disease. Biochem Soc Trans. 2014; 42(6): 1744-1751, doi: 10.1042/BST20140205, indexed in Pubmed: 25399600.

42. Radons J. The human HSP70 family of chaperones: where do we stand? Cell Stress Chaperones. 2016; 21(3): 379-404, doi: 10.1007/ s12192-016-0676-6, indexed in Pubmed: 26865365.

43. Mansilla MJ, Montalban X, Espejo C. Heat shock protein 70: roles in multiple sclerosis. Mol Med. 2012; 18: 1018-1028, doi: 10.2119/ molmed.2012.00119, indexed in Pubmed: 22669475.

44. Turturici G, Tinnirello R, Sconzo G, et al. Positive or negative involvement of heat shock proteins in multiple sclerosis pathogenesis: an overview. J Neuropathol Exp Neurol. 2014; 73(12): 1092-1106, doi: 10.1097/NEN.0000000000000136, indexed in Pubmed: 25383635.

45. Boiocchi C, Monti MC, Osera C, et al. Heat shock protein 70-hom gene polymorphism and protein expression in multiple sclerosis. J Neuroimmunol. 2016; 298: 189-193, doi: 10.1016/j.jneuroim.2016.07.011, indexed in Pubmed: 27609295.
46. Lechner P, Buck D, Sick L, et al. Serum heat shock protein 70 levels as a biomarker for inflammatory processes in multiple sclerosis. Mult Scler J Exp Transl Clin. 2018; 4(2): 2055217318767192, doi: 10.1177/2055217318767192, indexed in Pubmed: 29780609.

47. Khandia R, Munjal AK, Iqbal HMN, et al. Heat Shock Proteins: Therapeutic Perspectives in Inflammatory Disorders. Recent Pat Inflamm Allergy Drug Discov. 2017; 10(2): 94-104, doi: 10.2174/1872213X1 0666161213163301 , indexed in Pubmed: 27978789.

48. Matysiak M, Makosa B, Walczak A, et al. Patients with multiple sclerosis resisted to glucocorticoid therapy: abnormal expression of heat-shock protein 90 in glucocorticoid receptor complex. Mult Scler. 2008; 14(7): 919-926, doi: 10.1177/1352458508090666, indexed in Pubmed: 18573821.

49. Madeddu R, Farace C, Tolu P, et al. Cytoskeletal proteins in the cerebrospinal fluid as biomarker of multiple sclerosis. Neurol Sci. 2013; 34(2): 181-186, doi: 10.1007/s10072-012-0974-4, indexed in Pubmed: 22362332.

50. Gil-Perotin S, Castillo-Villalba J, Cubas-Nuñez L, et al. Combined Cerebrospinal Fluid Neurofilament Light Chain Protein and Chitinase-3 Like-1 Levels in Defining Disease Course and Prognosis in Multiple Sclerosis. Front Neurol. 2019; 10: 1008, doi: 10.3389/ fneur.2019.01008, indexed in Pubmed: 31608004.

51. Sellebjerg F, Royen L, Soelberg Sørensen P, et al. Prognostic value of cerebrospinal fluid neurofilament light chain and chitinase-3-like-1 in newly diagnosed patients with multiple sclerosis. Mult Scler. 2019; 25(11): 1444-1451, doi: 10.1177/1352458518794308, indexed in Pubmed: 30113249. 